\title{
Unusual II-I Phase Transition Behavior of Polybutene-1 Ionomers in the Presence of Long-chain Branch and Ionic Functional Groups
}

Chuanbin An, ${ }^{\dagger}$ Yahui Lou, ${ }^{\dagger}$ Yulian Li, ${ }^{\dagger}$ Bin Wang, ${ }^{\dagger}$ Li Pan, ${ }^{\dagger}$ Zhe Ma, ${ }^{\dagger, *}, 1$ and Yuesheng $\mathrm{Li}^{\dagger}, \dot{\dagger}$

$\dagger$ Tianjin Key Laboratory of Composite and Functional Materials, and School of Materials Science and Engineering, Tianjin University, Tianjin 300072, P. R. China

\$Collaborative Innovation Center of Chemical Science and Engineering (Tianjin), Tianjin 300072, P. R. China
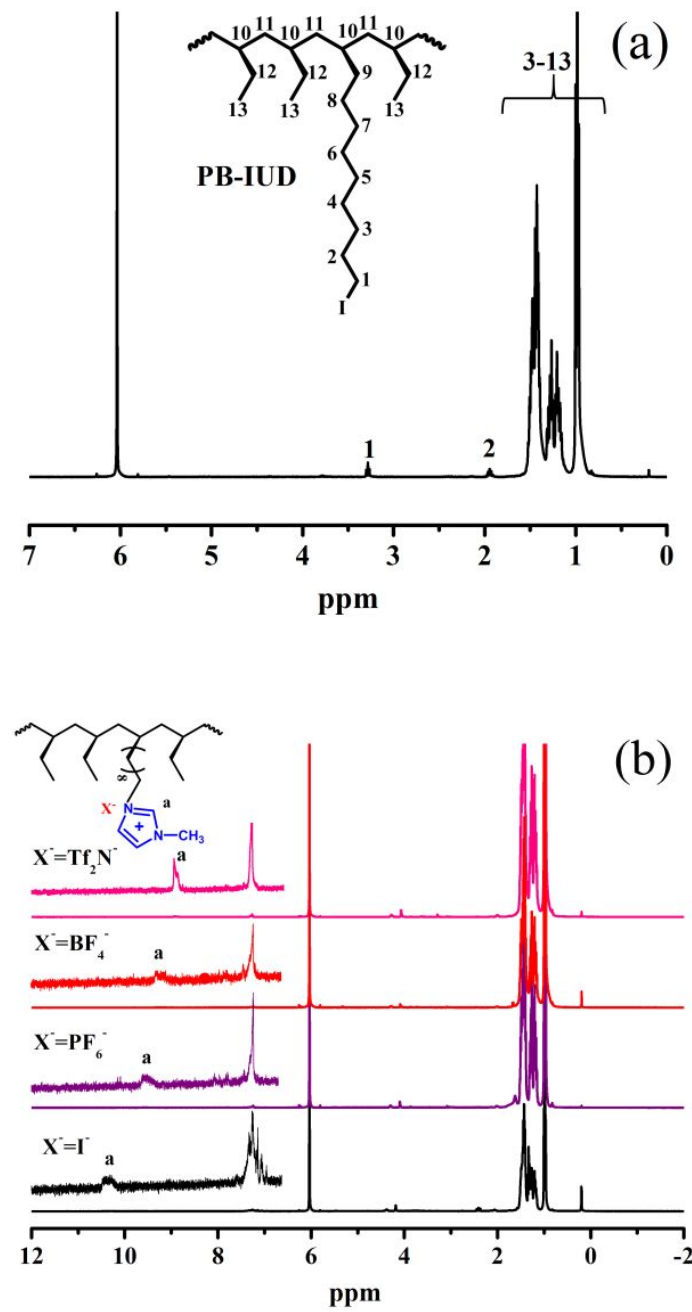

Figure S1. ${ }^{1} \mathrm{H}-\mathrm{NMR}$ spectra of homopolymer PB, copolymer PB-IUD, and ionomers with different counter-ions.

${ }^{1}$ Corresponding author: zhe.ma@tju.edu.cn 


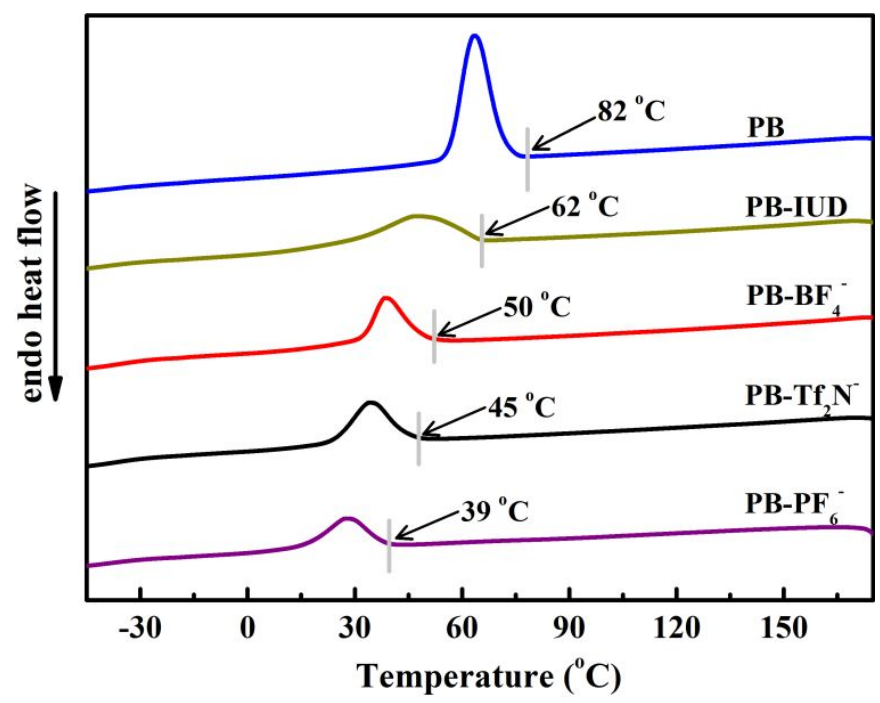

Figure S2. DSC cooling curves and the corresponding onset crystallization temperature of polymers studied in this work. The cooling rate was $10^{\circ} \mathrm{C} / \mathrm{min}$.

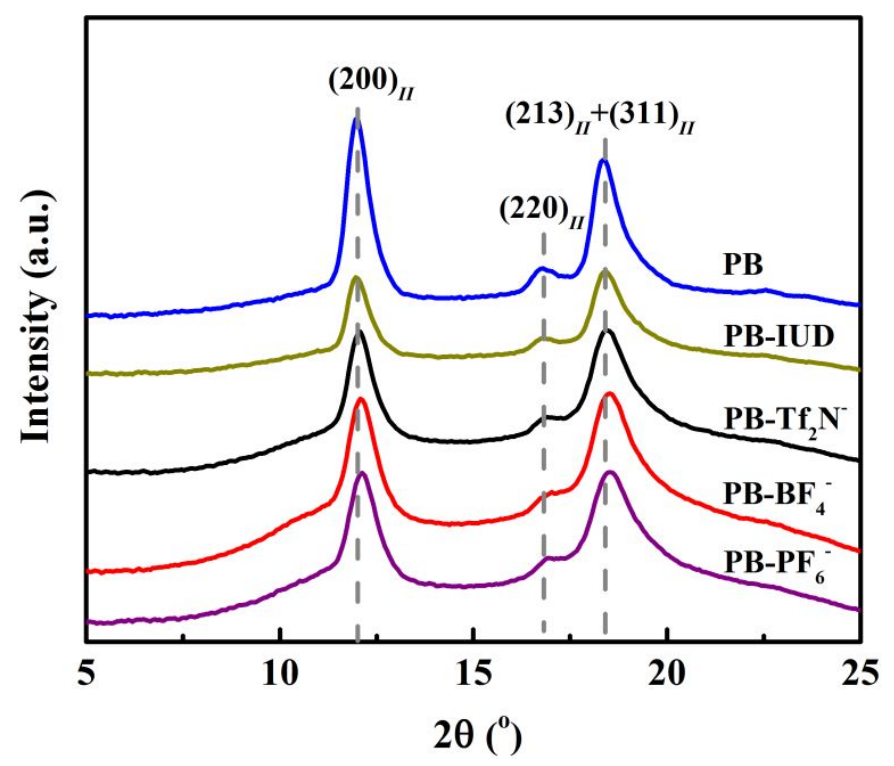

Figure S3. WAXD curves of samples isothermally crystallized at the temperature $5^{\circ} \mathrm{C}$ higher than the onset temperature of cooling crystallization. 


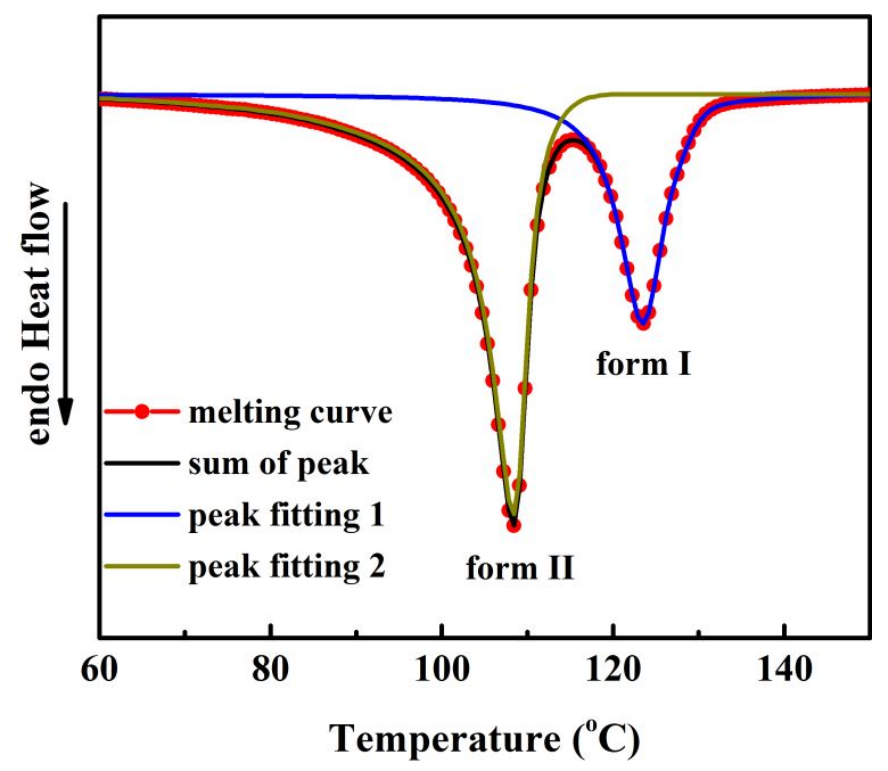

Figure S4. Fitting method to determine the melting entropies of residual form II and transformed form I.

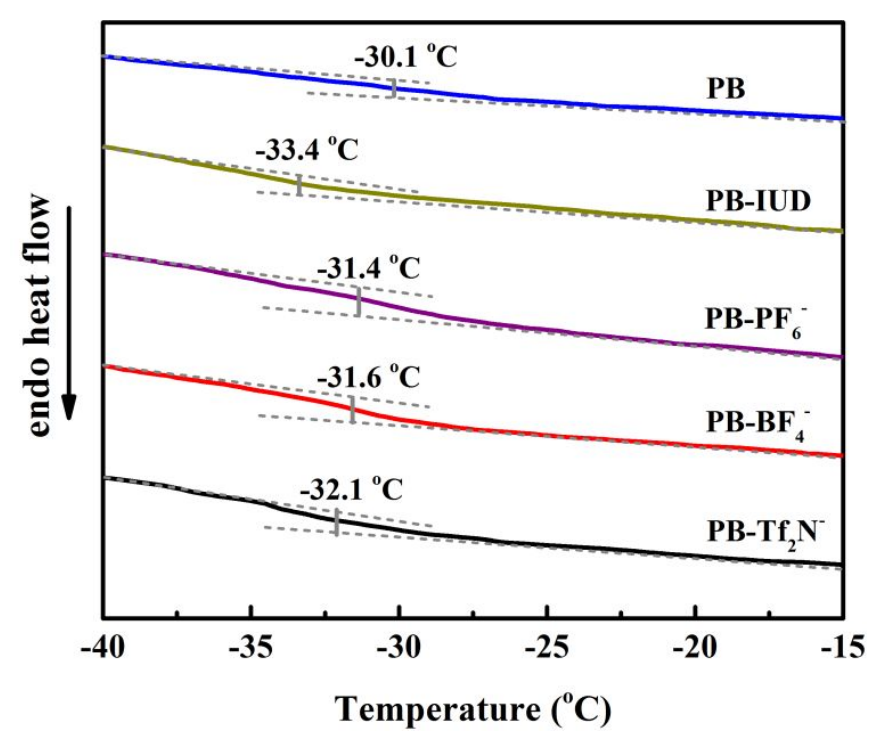

Figure S5. The DSC heating curves of homopolymer, copolymer, and ionomers to determine glass transition temperatures. 


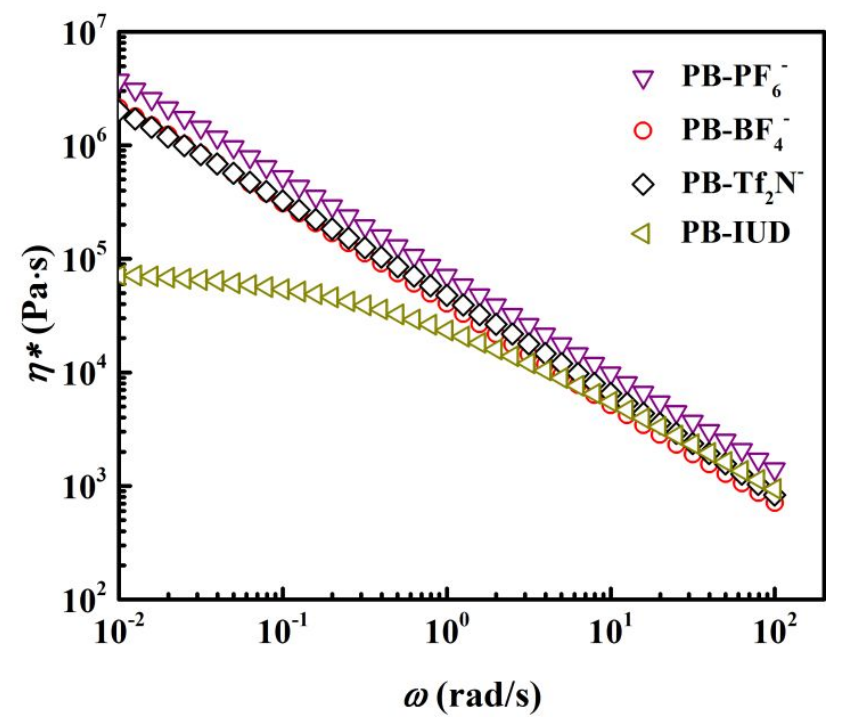

Figure S6. The melt viscosities of copolymer PB-IUD and ionomers measured determined by small amplitude oscillatory shear measurements performed at $140^{\circ} \mathrm{C}$. The choice of $140{ }^{\circ} \mathrm{C}$ is to avoid occurrence of crystallization during the rheological measurements. 